\title{
Jet performance in CMS
}

\section{Henning Kirschenmann*, on behalf of the CMS collaboration}

University of Hamburg, Institute of Experimental Physics

E-mail: henning.kirschenmanneuni-hamburg.de

The reconstruction and calibration of jets relies critically on the interplay between the tracking detectors, central calorimeters and forward calorimeters. Therefore, CMS makes use of the "Particle Flow" approach to optimally combine the information from the different subdetectors. A summary of the measurements of the jet energy calibration in CMS is presented, performed with data samples collected in proton-proton collisions at a center-of-mass energy of $8 \mathrm{TeV}$ corresponding to an integrated luminosity of $19.8 \mathrm{fb}^{-1}$. The final jet energy calibration is based on dijet, $\gamma+j e t$ and $\mathrm{Z}+$ jet events. The effect of pileup interactions and the state of the art mitigation techniques used in CMS are presented and we describe the main sources of uncertainty on the jet energy calibration.

The European Physical Society Conference on High Energy Physics 18-24 July, 2013

Stockholm, Sweden

\footnotetext{
* Speaker.
} 


\section{Introduction}

Jets are the experimental signatures of quarks and gluons produced in high-energy processes such as hard scattering of partons in proton-proton collisions. The jet energy corrections relate the energy of reconstructed jets - on average - to the true particle level energy, which is independent of the detector response. The detailed understanding of the jet energy scale is of crucial importance for many physics analyses.

The measurements discussed here have been performed by the Compact Muon Solenoid (CMS) collaboration at the CERN Large Hadron Collider (LHC). The central feature of the CMS apparatus is a superconducting solenoid, of $6 \mathrm{~m}$ internal diameter, providing a field of $3.8 \mathrm{~T}$. Within the field volume are a silicon pixel and strip tracker, a crystal electromagnetic calorimeter (ECAL), and a brass/scintillator hadron calorimeter (HCAL). Muons are measured in gas-ionization detectors embedded in the steel return yoke. Extensive forward calorimetry complements the coverage provided by the barrel and endcap detectors. A detailed account of the detector can be found in Ref. [1].

The techniques for the determination of the jet energy scale and transverse momentum resolution at CMS have been discussed in detail in Ref. [2]. In the following, we summarize the current status of pileup (PU) mitigation, as well as the latest jet energy corrections and the main sources of uncertainty on them.

\section{Jet reconstruction}

The jet energy corrections at CMS are derived for jets clustered using the anti- $k_{t}$ clustering algorithm with a radius parameter size of $R=0.5$. CMS uses the particle-flow event reconstruction $(\mathrm{PF})$ : It consists in reconstructing and identifying each single particle with an optimized combination of all subdetector information. The energy of photons is directly obtained from the ECAL measurement, corrected for zero-suppression effects. The energy of charged hadrons is determined from a combination of the track momentum and the corresponding ECAL and HCAL energy, corrected for zero-suppression effects, and calibrated for the nonlinear response of the calorimeters. Finally the energy of neutral hadrons is obtained from the corresponding calibrated ECAL and HCAL energy. As expected for the fragmentation of partons to jets, about $65 \%$ of the energy of PF-jets at central rapidity and $p_{T} \approx 100 \mathrm{GeV}$ is carried by charged hadrons, $25 \%$ by photons, and $10 \%$ by neutral hadrons. The study of the energy composition of PF-jets, i.e. the energy fractions of the different particle candidate classes, provides an important additional handle on the quality of the simulation of the detector and understanding of the PF algorithm. The differences in the observed energy fractions between data and simulation are below $1 \%$ in the central detector region as shown in Ref. [3].

\section{Factorized jet energy corrections}

The success of the CMS simulation in describing the jet properties (see Ref. [5, 6])allows its efficient use in a factorized approach to jet energy corrections (JEC) that CMS has adopted. The reference scale is that of generator jets, clustered from the four-momentum vectors of stable 


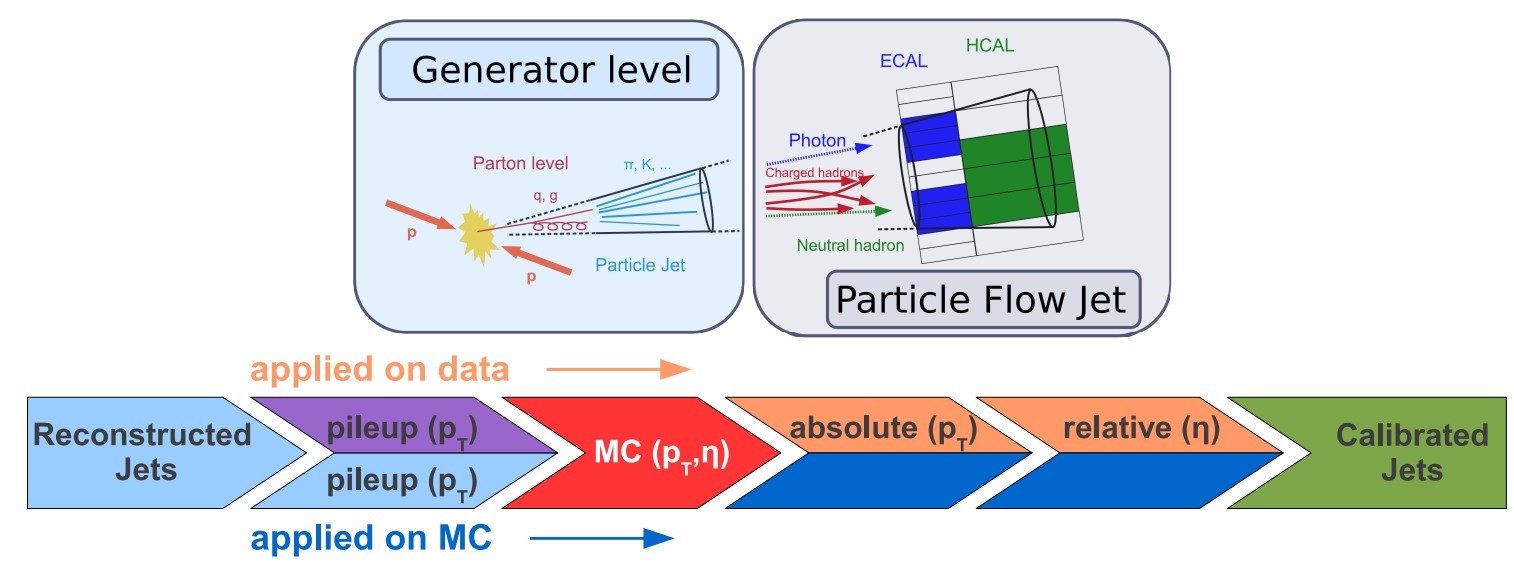

Figure 1: Factorized approach to jet energy corrections at CMS. Reference scale is that of generator jets.

particles (excluding neutrinos for PF) in the Monte Carlo simulation. On average, reconstructed jets are corrected back to this true particle level by applying the jet energy correction steps depicted in Fig. 1:

After the first level correction (L1), which subtracts the additional energy in the event induced by pileup effects (additional minimum bias events), the reconstructed jets are corrected to compensate for the non-linear response of the calorimeters (as a function of $p_{T}$ ) and variations of the response in $\eta$ (L2L3). The offset energy is measured in simulation and in data using the random cone method in zero bias events (compare Ref. [1]), the L2L3-corrections are derived from simulation. Subsequently, small residual corrections are applied which are based on measurements of the relative scale as a function of $\eta$ from dijet events and the absolute scale in the central detector region $(|\eta|<1.3)$ from $Z+$ jet and $\gamma+$ jet events (L2L3Residual).

\section{Pileup mitigation and pileup corrections}

An important complication for the JEC effort is the influence of additional energy depositions in the reconstructed jets not stemming from the initial hard scattering event. The additional energy caused by these soft $p p$-collisions is usually referred to as pileup. In the 2012 data taking, an average of 21 primary vertices were expected in addition to the hard scattering primary vertex. The average energy offset amounts to $\Delta p_{T} \approx 0.7 \mathrm{GeV} / N_{P V}$ for central PF-jets. A split-up of the offset energy into PF energy fractions as well as the offset energy measured in data and simulation for different $N_{P V}$ are shown in Fig. 2. A significant fraction of the PU energy can be attributed to charged hadrons from PU events and is mitigated by the charged hadron subtraction as described in section 4.1. The remaining offset energy is subtracted using the FastJet-based jet area method described in section 4.2.

\subsection{Charged hadron subtraction}

The particle-flow event reconstruction attempts to reconstruct individually each particle in the event, prior to the jet clustering. This allows for partial particle-by-particle pileup subtraction: The 

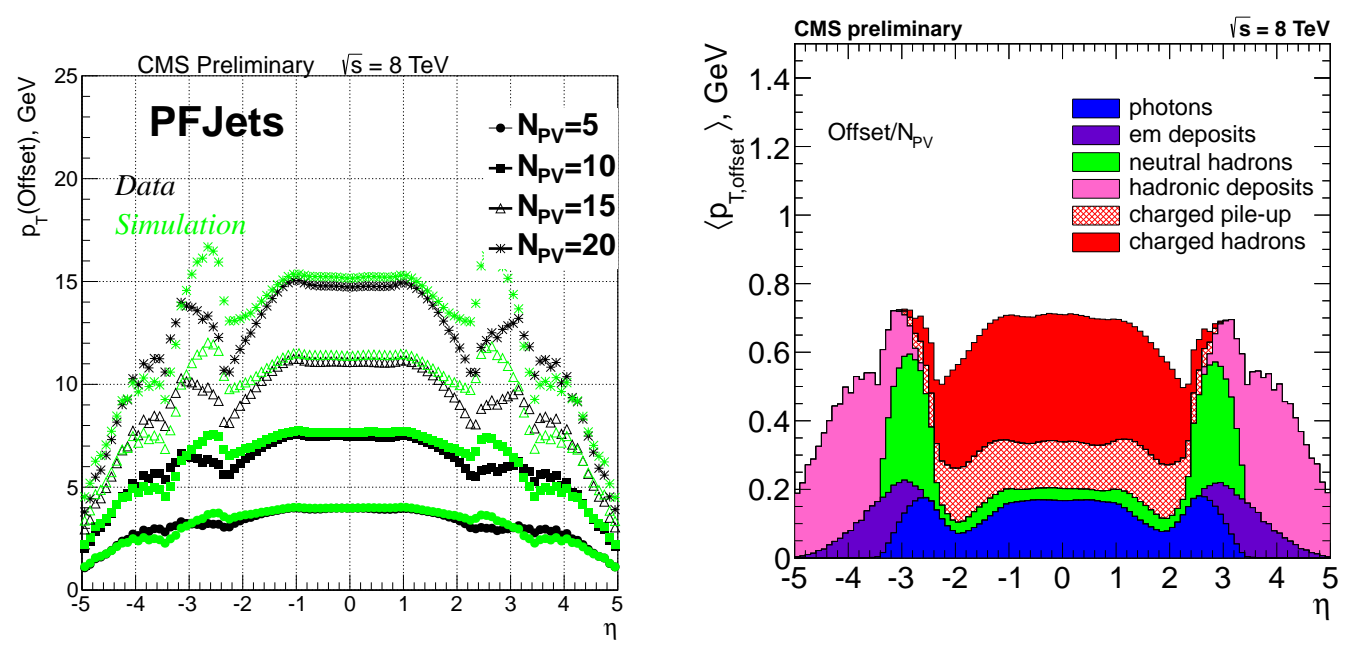

Figure 2: Left: Offset energy in data and simulation as a function of $\eta$ for different numbers of additional primary vertices. Right: Average offset energy (per additional primary vertex) split up into Particle Flow categories as a function $\eta$, taken from Ref. [3].

tracking information of charged hadrons is used to try to assign them to either the high- $p_{T}$ vertex or pileup vertices. The jet constituents which clearly belong to pileup vertices are removed from the jets. Those jet constituents without a clear association or an association to the high- $p_{T}$ vertex remain in the jet. As depicted in Fig. 2, this procedure accounts for roughly half of the offset energy from pileup in the tracker-covered region. Only the remaining offset energy has to be subtracted using the jet area method.

\subsection{Jet area method}

For each event, an average $p_{T}$ density $\rho$ per unit area is estimated which characterizes the soft jet activity and is a combination of the underlying event, the electronic noise, and the pileup. Following the jet area approach described in $[7,8], \rho$ and the jet-area A are used to calculate an $\eta$ and $p_{T}$-dependent correction factor (L1) to subtract the offset energy of individual jets on an eventby-event basis. The parameters are determined using two MC samples with identical hard-scatter events, with only one containing mixed-in pileup events. Residual differences in the offset energy measured using the random cone method in data and simulation (see Fig. 2) are taken into account.

\section{MC truth corrections}

The L2L3-corrections are based on simulation and correct the energy of the reconstructed jets such that it equals - on average - the energy of the jets at particle level. Simulated jet events, generated with PYTHIA6 [9], tune Z2* and processed through the full, GEANT4 [11] detector simulation are used for the derivation of these corrections. The generated and reconstructed jets are matched spatially and the transverse momentum of the reconstructed jet $p_{T}^{\text {reco }}$ and the response $p_{T}^{\text {reco }} / p_{T}^{\text {gen }}$ are determined in fine bins of $p_{T}^{\text {gen }}$ and $\eta^{\text {gen }}$. The correction factor is then determined as the inverse of the mean response as a function of $p_{T}^{\text {reco }}$ in fine $\eta$-bins. 

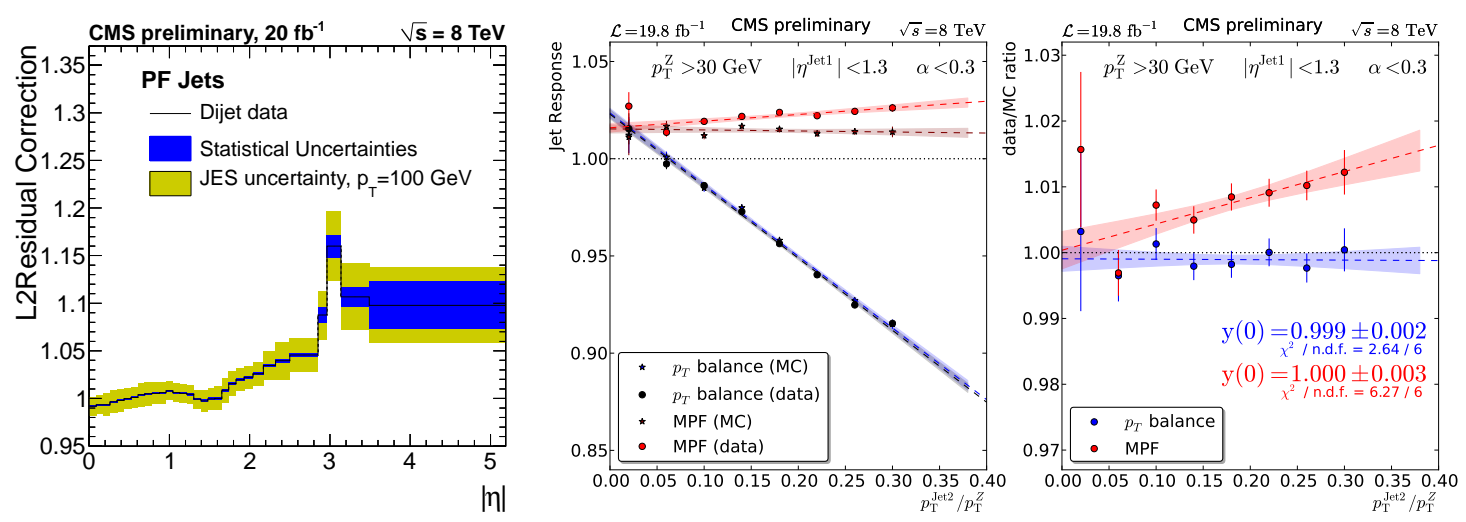

Figure 3: a.) Relative residual corrections as a function of $\eta$, determined from dijet events; b.) Extrapolation to perfect event topology for MPF and $p_{T}$ balance in data and MC for Z+jet events (as cross-check after applying the residual correction); c.) Extrapolation of data/MC ratio to perfect event topology (after applying the residual correction), taken from Ref. [4].

\section{Residual corrections from data-driven techniques}

The corrections from simulated data are the foundation of the jet energy correction chain in CMS. To complete the chain, these corrections are applied to data and simulated data in order to validate the jet energy scale. Response estimators from the $p_{T}$ balance and the MPF ${ }^{1}$ method (used extensively at [12], pioneered before at [13]) are used to determine the mean response in data and simulated data. Any small remaining differences observed in $\mathrm{Z} / \gamma+\mathrm{jet}$ and dijet events are explicitly corrected for in data in the L2L3Residual correction step which completes the previously discussed jet energy correction chain. A significant advantage of this approach is that biases inherent to datadriven methods are canceled to first order by determining only the ratio of data to simulation.

\subsection{Absolute scale from $\mathrm{Z}+$ jet and $\gamma+$ jet}

$Z+j e t$ and $\gamma+$ jet events provide a very clean signature with a well understood and precisely measured reference object balancing the jet. The jets are selected to be in the barrel region $(|\eta|<$ 1.3) and back-to-back to the reference object.

The response estimators are defined as

$$
\mathscr{R}_{\text {balance }}=\frac{p_{T}^{j e t}}{p_{T}^{\gamma, Z}}
$$

for the $p_{T}$-balance method and as

$$
\mathscr{R}_{\text {recoil }}=\mathscr{R}_{\gamma, \mathrm{Z}}+\frac{\overrightarrow{\mathscr{E}}_{\mathrm{T}} \cdot \vec{p}_{\mathrm{T}}^{\gamma, \mathrm{Z}}}{\left(\vec{p}_{\mathrm{T}}^{\gamma, \mathrm{Z}}\right)^{2}} \equiv R_{\mathrm{MPF}} \equiv \mathscr{R}_{\text {probe }}
$$

for the MPF-method, where $\vec{E}_{\mathrm{T}}$ is the missing transverse energy and $\mathscr{R}_{\gamma, \mathrm{Z}}$ the response of the reference photon or Z-boson. The idea underlying the MPF response estimator is that there is no

\footnotetext{
${ }^{1}$ Missing transverse energy projection fraction
} 

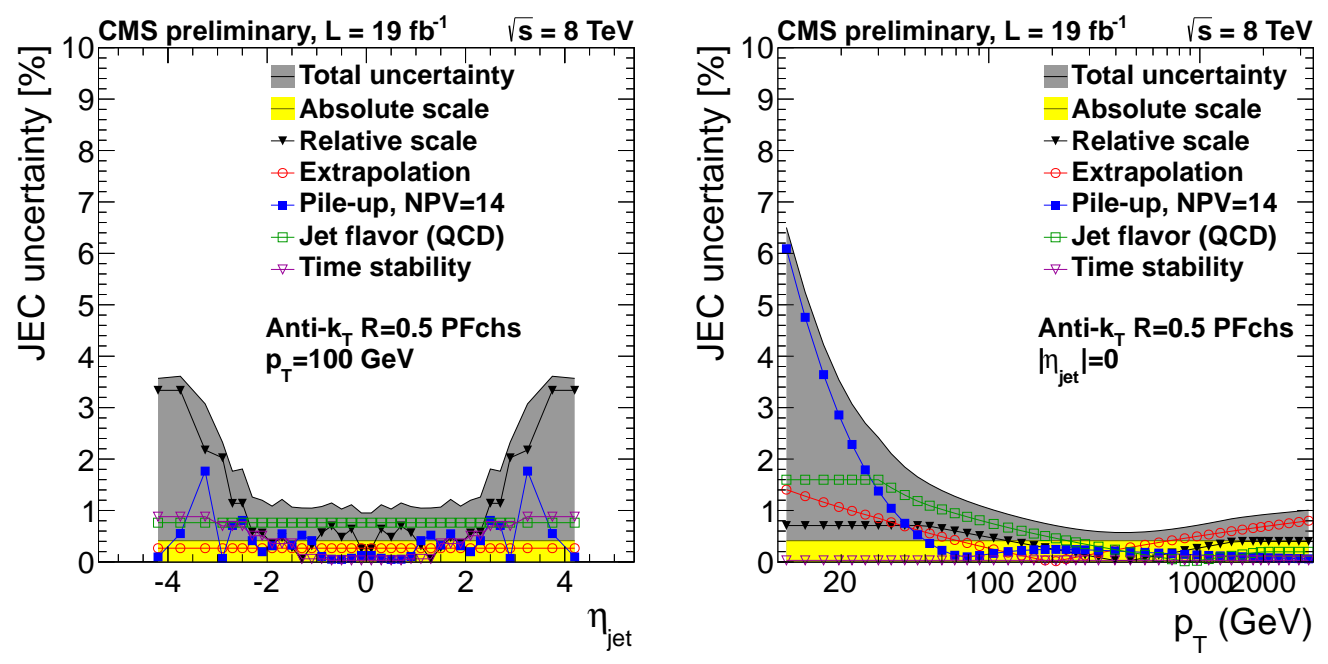

Figure 4: Jet energy scale uncertainties as a function of $p_{T}$ and $\eta$. Taken from Ref. [4]

intrinsic $\vec{E}_{\mathrm{T}}$ in such events and that the measured $\overrightarrow{\mathscr{E}}_{\mathrm{T}}$ is instead induced by mismeasurements of the hadronic recoil. The projection of $\vec{E}_{\mathrm{T}}$ along the reference object axis can then be used to yield a MPF response estimator.

In order to determine the energy scale as a function of $p_{T}$, the distributions of the previously defined response estimators $\mathscr{R}_{\text {balance }}$ and $\mathscr{R}_{\mathrm{MPF}}$ are evaluated. The mean of the estimated response is determined in bins of $p_{T}$ for different values of the relative second jet $p_{T}, \alpha=p_{T}^{\text {Jet2 }} / \vec{p}_{\mathrm{T}}^{\gamma, \mathrm{Z}}$. The ratio of data to simulation of $\mathscr{R}_{\text {balance }}$ and $\mathscr{R}_{\mathrm{MPF}}$ is extrapolated to zero additional event activity (corresponding to $\alpha=0$ ) to suppress the influence of soft radiation on the results. An example of such an extrapolation is shown in Fig. 3.b and illustrates the significantly reduced dependence of $\mathscr{R}_{\text {MPF }}$ on the radiation modeling in simulation with respect to the $p_{T}$ balance method. As a crosscheck after applying the resulting residual correction, the ratio of data to simulation as a function of the cut on additional event activity is depicted in Fig. 3.c and shows closure of the correction.

\subsection{Relative scale from dijet events}

With the absolute scale being verified in the central detector region, the missing component is the relative scale. This is determined using dijet events which provide a large number of events and a high $p_{T}$-reach. The two leading jets are required to be back-to-back in the azimuthal angle $\varphi$ and at least one of the jets is required to be in the central $(|\eta|<1.3)$ detector region.

With this selection, the energy scale can be constrained relative to the central detector region by response estimators, i.e. the relative response

$$
\mathscr{R}_{\mathrm{rel}}\left(\eta^{\text {probe }}, p_{\mathrm{T}}^{\mathrm{ave}}\right)=\frac{1+\langle\mathscr{A}\rangle}{1-\langle\mathscr{A}\rangle}, \text { with } \mathscr{A}=\frac{p_{T}^{\text {probe }}-p_{T}^{\text {barrel }}}{p_{T}^{\text {probe }}+p_{T}^{\text {barrel }}}
$$

and $\mathscr{R}_{\text {MPF }}$ as before. As depicted in Fig. 3.a, these relative differences are very small in the central detector region and below $5 \%$ in the region up to $|\eta|<2.5$. 


\section{Systematic uncertainties}

As a result of the factorized calibration procedure outlined above, the jet energy scale at CMS is known to the percent level over a wide range of the available phase space (see Fig. 4) and as precise as $1 \%$ at $200 \mathrm{GeV}$ for a central jet. The systematic uncertainties are dominated by pileup uncertainties at low $p_{T}$. For the intermediate $p_{T}$-region, the jet flavor uncertainty (determined from PYTHIA/HERWIG++ [10] response differences of individual flavors) becomes dominant and for high $p_{T}$, the extrapolation uncertainties (taking into account variations of the single particle response) become dominant. For the more forward detector region, the relative scale uncertainty from the dijet relative residual corrections are dominant. The full split-up of uncertainty sources is available for analyses, thus preserving the correlations e.g. for combinations of results with other experiments.

\section{Conclusions}

We presented the current status of jet energy corrections at CMS. The influence of pileup and the current pileup mitigation techniques adopted by CMS in the context of jet energy corrections were discussed. The improved understanding of systematic uncertainties associated with the jet energy corrections allows many physics analyses to improve on their JEC-related systematic uncertainties, in turn leading to more precise results in measurements involving jets.

\section{References}

[1] The CMS Collaboration, The CMS experiment at the CERN LHC, JINST 3 S08004 doi:10.1088/1748-0221/3/08/S08004

[2] The CMS collaboration, Determination of jet energy calibration and transverse momentum resolution in CMS, JINST 6 P11002 doi:10.1088/1748-0221/6/11/P11002

[3] The CMS Collaboration, Jet Energy Corrections and Uncertainties. Detector Performance Plots for 2012, CMS Detector Performance Summaries, DP-2012-012

[4] The CMS Collaboration, Jet Energy Corrections and Uncertainties with $19.8 \mathrm{fb}^{-1}$, CMS Detector Performance Summaries DP-2013-033

[5] The CMS Collaboration, Jet Performance in pp Collisions at 7 TeV, CMS-PAS JME-10-003

[6] The CMS Collaboration, Performance of quark/gluon discrimination in $8 \mathrm{TeV}$ pp data, CMS-PAS JME-13-002

[7] M. Cacciari and G.P. Salam, Pileup subtraction using jet areas, Phys. Lett. B 659119

[8] M. Cacciari, G. P. Salam and G. Soyez, FastJet user manual, Eur. Phys. J. C 721896

[9] T. Sjöstrand, S. Mrenna, and P. Skands, PYTHIA 6.4 physics and manual, JHEP 05:026

[10] M. Bahr et al., Herwig++ physics and manual, Eur. Phys. J. C 58639

[11] S. Agostinelli et al., Geant 4 - A Simulation Toolkit, Nucl. Inst. Meth. A 506 250-303

[12] D0 collaboration, Determination of the absolute jet energy scale in the DO calorimeters, $N$ ucl. Instrum. Meth. A 424352

[13] CDF Collaboration, Dijet angular distribution in p p collisions at $\sqrt{s}=1.8 T e V$, Phys. Rev. Lett. 69 2896 